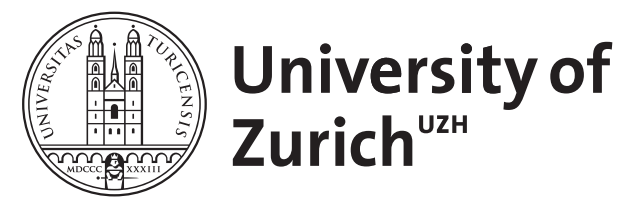

\title{
Unity in the concept of function
}

\author{
Steiner, Christian
}

\begin{abstract}
This essay argues that neither the etiological nor the dispositional account of functions conforms to the actual practice by which functions are ascribed in biology. Philip Kitcher's account, which unifies what is common to both accounts, is assessed against what biologists are actually doing when they ascribe functions. Two problems of Kitcher's account are identified: it is too liberal and it tends to circularity, insofar as it presupposes teleological notions. Finally, an alternative account of functions is provided by characterizing the system of sentences that report natural history.
\end{abstract}

DOI: https://doi.org/10.1515/cpt-2009-9408

Posted at the Zurich Open Repository and Archive, University of Zurich ZORA URL: https://doi.org/10.5167/uzh-54296

Journal Article

Accepted Version

Originally published at:

Steiner, Christian (2009). Unity in the concept of function. Conceptus, 38(94):91-106.

DOI: https://doi.org/10.1515/cpt-2009-9408 


\title{
Unity in the Concept of Function
}

\author{
Christian Steiner* \\ University of Zurich
}

December 1, 2010

\begin{abstract}
This essay argues that neither the etiological nor the dispositional account of functions conforms to the actual practice by which functions are ascribed in biology. Philip Kitcher's account, which unifies what is common to both accounts, is assessed against what biologists are actually doing when they ascribe functions. Two problems of Kitcher's account are identified: it is too liberal and it tends to circularity, insofar as it presupposes teleological notions. Finally, an alternative account of functions is provided by characterizing the system of sentences that report natural history.
\end{abstract}

\section{Biological Functions}

Functions are used both in order to explain what something is and why it is doing what it does, by citing one of its effects. This sort of explanation is unproblematic in the case of artefacts, for artefacts are intentionally designed to do something. Functions are also attributed, however, to natural objects, and biologists have found it difficult, even undesirable, to eliminate this form of explanation from their discussions of biological phenomena (Allen and Bekoff 1995, 610). Thus, we are in need of an account of natural teleology. We have to answer the question of how it is possible to explain something by citing its function, i.e., one of its effects.

There are two dominant positions in the debate on biological functions, providing two different answers to this question. On the one hand, the etiological account, first elaborated by Wright (1973), understands functions as effects of objects which contributed to the presence or persistence of those

*christian.steiner@philos.uzh.ch 
objects. More specifically, the most prominent versions of this account understand functions as selected effects (cf. Millikan 1984; Neander 1991; Griffiths 1993; Godfrey-Smith 1994; Allen and Bekoff 1995). According to these philosophers, functional explanations are unproblematic because they refer to what was initially responsible for the existence of some object. On the other hand, the dispositional account, first elaborated by Cummins (1975), understands functions as effects which contribute to more complex dispositions of the relevant containing system. Since function ascriptions are interestrelative, according to this account, to explain something by reference to its function is to refer to its causal role in producing other things that interest us. Hence, functional explanations seem innocuous.

Although the question as to which account should be favoured was initially hotly contested, today most commentators agree that these two accounts pick out two different notions of function, which should be kept apart (cf. Godfrey-Smith 1999; Millikan 2002; McLaughlin 2001). In contrast, Philip Kitcher argues that we are in need of a unified account of function, for whilst both accounts seem to relate to functions as they are attributed in biology, each of them captures only some aspect of how they are ascribed in particular biological fields. By paying attention to the different function ascription practices in biology, Kitcher wants to provide a unified account of functions.

I am sympathetic to Kitcher's sensitivity to the actual practices of ascribing functions in biology. It seems to me that the debate would substantially profit from similar endeavours to analyze what biologists are actually doing when they are ascribing functions to the structures and behaviours of organisms. Therefore, I wish to assess Kitcher's unified account and discuss an alternative account which follows him in paying attention to biological practice.

I start by establishing the need for a unified account. I do so by considering different ways of reacting to the fact that there are these two conceptions of function. As will be shown, there are only two options available for someone who takes the different biological practices of ascribing functions seriously: one has to provide either an account that unifies what is common to those two conceptions of function, or an alternative account which does not reduce teleological to non-teleological vocabulary. I present Kitcher's account as the most promising version of a unified reductionist account in section 3, and discuss two possible problems for his account in section 4 . Finally, I sketch an alternative account of biological function. 


\section{Two Conceptions of Function}

There are two conceptions of biological function acknowledged in the current debate: the etiological and the dispositional accounts. According to the most prominent version of the former, some item $\mathrm{X}$ has the function of doing $\mathrm{F}$ if it has been selected for doing F. According to the latter, functions are effects that contribute to more complex dispositions of a containing system.

Proponents of the etiological account of biological functions think that their account has the following merits: $(\alpha)$ it accounts for the distinction between a trait's proper function and its merely accidental effects, $(\beta)$ it accounts for the possibility of a trait malfunctioning, and $(\gamma)$ it maintains a realist view in respect of functions.

However, there are some well-known issues with this account: $(\chi)$ since the first occurrence of a trait has not been selected for, the initial effect of that trait which has led to the recurrence of a similar trait in the descendant organisms cannot be the function of the original trait, $(\psi)$ this account cannot differentiate between functions and vestiges, and $(\omega)$ according to this account, but contrary to our practice, we would have to ascribe functions to whole organisms (McLaughlin 2001, 99).

The dispositional account's merits and disadvantages mirror the etiological account's disadvantages and merits: it is not committed to $(\chi),(\psi)$, and $(\omega)$, but cannot account for $(\alpha),(\beta)$, and $(\gamma)$. This account is not committed to the counter-intuitive claim that an ancestor's behaviour does not have a function, although it behaves in exactly the same way as its descendants do. Neither does it imply that useless traits, which were once selected, should be considered as having a function. Nor does it entail that we should ascribe a function to whole organisms. It, therefore, accords with ordinary use. Yet the dispositional account cannot distinguish between a trait's proper behaviour and its accidental effects. Neither can it account for a biological trait's malfunctioning. Since this account cannot maintain a realist position with regard to function, functions seem to be imposed on nature by us.

How are we to react to there being those two conceptions of function in light of their problems? There are four options available:

1. Claim that one of the two accounts is right (and reformulate it in order to avoid its problems). ${ }^{1}$

\footnotetext{
1 Recently two accounts have been developed that try to improve the etiological and dispositional account, respectively, by appeal to "self-reproduction". According to McLaughlin's version of the etiological account (2001), something has a function if it contributed to its own maintenance over time. According to Weber's version of the dispositional account (2005), a capacity is a function if it is part of a coherent system of capacities. A
} 
2. Claim that these two accounts should be kept apart because they serve different purposes.

3. Provide a unified reductionist account.

4. Develop an alternative account of biological functions according to which functions are not be analysed in non-teleological vocabulary.

Which options are available to a philosopher who takes current biological practices of ascribing functions seriously?

We have to rebut option (1) if we consider the practices in e.g., both behavioral ecology and developmental biology, given that function ascriptions in such fields as developmental biology often appear to make no reference to evolution or selection, whereas function ascriptions in such fields as behavioral ecology do not depend on the contribution of a behavior to a more complex disposition.

What about option (2)? Godfrey-Smith (1999), for example, argues for this position. He claims that etiological and dispositional accounts relate two different concepts and that there is no strong reason for using the same word for both kinds of function. We have to rebut this option, however, if we consider an account based on that proposal as being an explanation of how functions are attributed in biology. For, irrespective of the particular field, function attributions in biology do not seem to be interest-relative (cf. Weber 2005, 191). Thus, the dispositional account, which claims that function ascriptions depend on the investigator's interest, cannot cover the function-attribution practice of any of the fields in biology. Since the etiological account does not cover all fields, the practice of ascribing functions in some fields would still be unexplained if we accepted a pluralistic view of functions.

What then of options (3) and (4)? Option (4) aims to account for functions without trying to reduce teleological to non-teleological vocabulary. It is not obvious whether such an account would conflict with the different practices of function ascription in the different areas of biology - although one might suspect it might. In any event, such an account would not be very fashionable since there seems to be a general agreement among commentators that a reductive program is the only way to treat functions. I shall therefore postpone the discussion of this option to section 5, in which I explicate such an account, and consider, now, option (3).

system of capacities is coherent if each capacity contributes to another capacity. Such a system of capacities explains how an organism can self-reproduce. Both accounts have problems, however, ascribing functions to reproductive organs, as reproductive organs do not contribute to the self-reproduction of an organism. 
If a unified account of functions could be provided, it would both be sensitive to the pluralistic practice of ascribing functions in biology and be able to account for what is common to these different ascriptions. On the assumption that pluralism is untenable, it is the only reductive account available to the philosopher who takes seriously the current practices of function ascriptions in the different areas of biology.

Having established the need for a unified account of function, I now want to present and discuss Kitcher's version of such an account.

\section{Kitcher's Unified Account of Function}

In "Function and Design", Kitcher proposes a unified account of function that is general not only insofar as it pays attention to the pluralistic functionattribution practice in biology, but also in virtue of it covering functions of both artefacts and organisms.

According to Kitcher, the key notion for understanding the concept of a function is design. Artefacts have functions because they are intentionally designed to do something. For instance, the function of a microwave oven is to heat meals because that is what it is designed to do. Likewise, there is a direct link between the functions of organisms and their design. PreDarwinian accounts of function presupposed divine intentions in order to explain the design of organisms. According to post-Darwinian accounts, however, "we can think of design without a designer" (Kitcher 2003, 160). The source of natural design lies in the action of natural selection: what a given organ is designed to do is that for which it was selected.

Up to this point, Kitcher's account seems to correspond to the etiological account. What the functions of artefacts and of organisms have in common is that they both depend on design. Nonetheless, according to Kitcher, this dependence can be more or less direct. So, for example, it is possible that a person who is making a machine forgets to fit a certain component that plays a crucial part in the machine's proper functioning. If something accidentally becomes lodged in the right position, however, and fulfils the job of the forgotten component, then we can ascribe a function to it even though that thing is not intentionally designed to do what it is now doing. Equally, a biological trait may respond to a selection pressure, and thus be attributed a function, even though it is not designed to do what it does because it was not selected for doing that. In such cases, functions are attributed to entities that are not designed to do something, but rather stand in an indirect relation to design: they fulfil tasks that are imposed on them by the design of the whole. 
Now, one could worry that the unity arising from the notion of design might reveal itself as rather superficial, insofar as one could argue that we attribute two different conceptions of design to artefacts and to organisms. We would also need a unified account of design. Thus, it seems as if this notion cannot cover the different ascriptions of functions.

Yet, notwithstanding the mentioning of 'design' in the title of his paper, on a more careful reading of "Function and Design", it seems to me that this notion does not play such a crucial part in Kitcher's account of function. More recently, Kitcher (2011) claims that the notion of a problem background is what really constitutes the unity in the concept of function: something has a function if it stands in a context involving a problem background and responds as a solution to those problems. In the case of artefactual functions, the inventor of an artefact faces a problem, and the wish to solve it serves as the basis of the artefact's function. In the biological case, the problems are generated by selection pressures arising from the interaction between the organism's constitution and its environment.

The notion of a problem background unites the two conceptions of biological function. For, on the one hand, an organism only has a problem background if it faces selection pressures, whilst on the other, biologists in certain areas attribute functions without knowing the exact evolutionary history of a trait of an organism. Instead, they rely on the problem background for the organism in question in order to pick out the contributions its traits make to solving some problem.

In summary, Kitcher's unified account of function captures the intuitions behind both conceptions. As per the etiological account, this account emphasises the role of natural selection for ascribing functions, insofar as a problem background is constituted only by selection pressures. Akin to the dispositional account, this account does not demand that a trait's contribution to survival or reproduction be one that has been naturally selected for, in order to be reckoned as its function; rather, the contribution has to be seen as responding to the organism's problem background.

\section{Two Problems for Kitcher's Account}

In section 2, I considered how one might react to there being two conceptions discussed in the current debate on biological function. I excluded two ways of reacting: claiming that only one account is right, and claiming that the two conceptions should be kept apart because they serve two different purposes. After presenting Kitcher's unified account, I would now like to ask whether this account is really available to the philosopher who takes the biological 
practices of ascribing functions seriously, and whether this account really explains what it is supposed to explain. I shall present two reasons why both questions might be answered in the negative.

Concerning the first question, Kitcher's account may well fail to conform to the biological practice of ascribing functions, insofar as his account might be considered too liberal. As with the dispositional account, this account makes every contribution of a trait to the survival or reproduction of the organism look to be its function, even if the contribution happens accidentally. I am not sure, though, whether biologists do indeed recognise such contributions as functions.

Here is an example of such an accidental contribution. This example is the closest analog to Kitcher's own example of an indirect artefactual function. As stated earlier, it is Kitcher's view that we can attribute a function to an artefact's forgotten component which accidentally becomes lodged in the right position and, thus, fulfils a job. Similarly, even the accidental contribution of a trait to the survival of the organism can, according to his account, be recognised as its function. However, in the case of biological function, we would have to attribute a function even to a disease were it to contribute to the overall fitness of the organism. Indeed, diseases need not have uniformly negative effects on the fitness of organisms. As Jarosz and Davelos (2006) indicate, fungal endophytes of the genus Myriogenospora reduce both the growth and reproduction of plants but also provide protection from herbivores. Thus, grasses infected with fungal endophytes of this genus have slightly increased fitness relative to uninfected plants under high herbivore pressures. Yet, although phytologists notice the positive effects certain fungal endophytes have on their hosts, they do not seem to consider them as functions for these hosts - as they ought, according to Kitcher's account. ${ }^{2}$

Concerning the second question, as to whether or not this account explains what it is supposed to explain, I would like to suggest that Kitcher's account is circular insofar as it presupposes teleological notions. The notion of a problem background seems to have application only for things that aim at being in certain conditions. Thus we can attribute this notion only if we already understand that to which it applies as something that should be a certain way. But to understand something in this way is to understand it as having a telos - yet that is exactly what Kitcher's account of function is supposed to provide.

\footnotetext{
2 To be sure, under certain conditions, allowing some fungi to thrive on the plant could be a function. The infection of a plant with some fungal endophytes would only count as something the plant allows, however, if we could distinguish cases when the plant fights the infection from those when it remains passive. Yet, the cases Jarosz and Davelos (2006) report do not leave room for such a distinction.
} 
Let me illustrate my claims with a quotation from Kitcher's "Function and Design". He writes:

Holding the principal features of the environment fixed, we can ask what selective pressures are imposed on members of a group of organisms. In posing such questions we suppose that some of the general properties of the organisms do not vary and consider the obstacles that must be overcome if organisms with those general properties are to survive and reproduce in environments of the type that interests us. (2003, 161, my emphasis)

We ascribe functions by identifying contributions to the overcoming of obstacles and we identify obstacles by considering what it is that organisms of a certain constitution, in a certain environment, have to overcome if they are to survive and reproduce. Now, how are we to understand the if-clause of the last sentence? We can understand it either as simply hypothetical thinking or as what those organisms are supposed to do. I suggest that only if we understand it in the latter sense, can we identify the need to survive and to reproduce as that which constitutes real pressures imposed on organisms. Since those needs can only constitute real pressures for organisms if they are supposed to obtain, organisms are supposed to have those needs. To say that organisms need to reproduce, to survive long enough, and to be fertile, is, however, to say that organisms should do certain things. Yet an account of biological function is supposed to explain how we are to understand that organisms should do certain things. In this respect, Kitcher's account shows a certain degree of circularity.

\section{An Alternative Account of Function}

What might an alternative account of biological function look like? I would now like to sketch such an account. In contrast to the three accounts discussed so far, an alternative account of biological function need not consist in a reduction of teleological to non-teleological vocabulary. Instead, the concept of a biological function can be shown to relate to a form of description of events. This concept is then elucidated by showing how such a particular form of description differs from other forms of description.

Let me explain. The debate about how to understand biological functions is dominated by the view that to explain the concept of a function is to say something about the content of a function-ascribing sentence. According to the standard view, an analysis of the concept of a function consists in specifying necessary and sufficient conditions for something to be reckoned as 
falling under this concept. In contrast, the alternative project here proposed is to characterize a system of sentences to which a sentence joining an item $x$ and a predicate $\mathrm{F}$ belongs, if it is true that $x$ has the function of doing F. Therefore, this alternative account proceeds by analysing a system of sentences which do not contain explicit ascriptions of function.

I now look at cases of implicit function-ascriptions in order to show how such an alternative account might explain the concept of a biological function. The example I discuss is from the same paper Kitcher uses in order to illustrate his own account. The following account reports the natural history of spiders of the species Nephila clavipes:

Spiderlings overwinter in the egg sac for six to eight months, emerging under favourable conditions in March. [.... After two or three weeks] spiderlings [...] build and inhabit individual orbs on which prey are captured. In early July, males begin to mature adulthood and abandon their orbs in search of females. Males compete for a position proximal to the female, near the hub of the web. $[\ldots]$ Copulations occur primarily during the two-day period immediately following the female's final moult $[\ldots]$. During the next few months females lay up to three egg clutches, each containing several hundred eggs. [...] Second instars, capable of silk production, overwinter within the egg sac without the benefit of the mother's protection. (Christenson and Wenzl 1980, 1110)

What functions are implicitly attributed in this account? Let me make explicit some of the functions to be found. The egg sac has the function of protecting the spiderlings in overwintering. The building and inhabiting of orbs has the function of capturing prey. The males' abandoning of their orbs has the function of finding females to mate. The males' competing behaviour has the function of copulating with a female, and copulation, obviously, has the function of reproduction.

Now, how did I identify these different functions? Two principles seem to be at work in identifying them. The first principle is that whenever this account reports that spiders do $\mathrm{F}$ in, on or with some item $x$, then doing $\mathrm{F}$ is the function of $x$. Ascribing a function to the egg sac, for instance, accords with this principle. The second principle seems to be that contributing to the behaviour mentioned in a subsequent sentence is the function of the behaviour mentioned in a previous sentence. Ascribing the function of copulating to the competing behaviour of males, for instance, accords with this principle. Thus, the sentences reporting this natural history correspond to one of two schemata: they have either the form 'The $\mathrm{S}$ does $\mathrm{F}$ in, on or with $x$ ' or 'First, the $\mathrm{S}$ does $\mathrm{F}$, and then it does G'. Functions that are expressed in 
sentences corresponding to the first schema are attributed to structures or organs. Functions of the second kind, on the other hand, are attributed to behaviour. Yet, functions of the latter kind are prior to functions of the former kind, since instances of the first schema are embedded in the second schema and, therefore, depend on there being functions of the latter kind.

As the second schema orders its sentences, these sentences form a system. The system in question is temporally ordered, such that each sentence within this system reports what members of this species do at a certain stage in their lives. As can be seen in the example, such a system starts and ends with a sentence reporting the behaviour of a new generation. Thus, the system is characterizable as a cyclical system: what is reported in this system happens again and again in the same order. However, the sentences that are part of this system do not only relate to each other in virtue of depicting what happens earlier or later; they depend on each other, insofar as each sentence is only intelligible and thus true as part of the whole system.

Consider a cyclical system for which this is not true, for instance, the Carbon-Nitrogen-Oxygen cycle (the CNO-cycle), which is the dominant source of energy in stars more massive than the sun. Although the sentences describing the CNO-cycle form a cyclical system, these sentences do not presuppose the system in order to be intelligible. So, for example, a sentence describing the transformation from nitrogen to oxygen $\left({ }_{7}^{14} N+{ }_{1}^{1} H \rightarrow{ }_{8}^{15} O+\gamma\right)$ is intelligible and true independently of the cycle. In contrast, we could not understand a sentence such as "Copulations occur primarily during the twoday period immediately following the female's final moult", were there not a system in the background reporting the natural history of the species in question. For the reference to moult and reproduction would be void.

In summary, the sentences reporting the natural history of a species form a cyclical system on which their intelligibility and truth depend. We can therefore characterize such a cyclical system as a holistic system. ${ }^{3}$

After characterizing the system of sentences reporting the natural history of a species, I am now in a position to give an account of biological function. If the observation is correct that biologists, in expanding our knowledge, aim at contributing true sentences to cyclical and holistic systems, then to ascribe a particular function to a structure or behaviour is to claim that any sentence joining the structure or behaviour with a subject belongs to such a system. In other words, the question whether something has a function asks whether

3 According to Weber (2005), functions are capacities that form a coherent system. He characterizes such a system as both cyclical and holistic. Whereas I describe a system of sentences, he holds that it is the capacities themselves which constitute such a system. Therefore, his account - unlike my own - seems to have problems ascribing functions to reproductive organs. 
a sentence mentioning this structure or behaviour is part of a cyclical and holistic system of sentences. To ask what function a structure or behaviour has is to ask for the position of the relevant sentence within such a system.

\section{Conclusion}

The account of biological function presented in this paper differs from standard accounts in several respects. First, it does not attempt to reduce teleological to non-teleological vocabulary, as it has not been established that teleological explanations are more complex mechanical explanations. Second and relatedly, this account does not specify the necessary and sufficient conditions for something to fall under the concept of a function. Instead, I have offered an account of biological functions by reflecting on how biological facts are represented. Sentences reporting biological phenomena form a special kind of system. Such a system of sentences is special in being both cyclical and holistic. In order to give a full account of biological functions, one would have to say more to characterize the system of sentences in question. ${ }^{4}$ However, if my considerations are right, then ascribing a function to a structure or behaviour is claiming that a sentence which joins this structure or behaviour with its subject is part of such a system. ${ }^{5}$

\section{References}

Allen, C. and M. Bekoff (1995). Biological Function, Adaptation, and Natural Design. Philosophy of Science 62(4), 609-622.

Christenson, T. and P. Wenzl (1980). Egg-laying of the Golden Silk Spider, Nephila clavipes L. (Araneae, Araneidae): Functional Analysis of the Egg Sac. Animal Behaviour 28(4), 1110 - 1118.

Cummins, R. (1975). Functional Analysis. The Journal of Philosophy 72(20), 741-765.

Godfrey-Smith, P. (1994). A Modern History Theory of Functions. Noûs 28(3), 344-362.

4 So, for example, Thompson (2008) argues that sentences constituting such a system exhibit a non-Fregean generality. Relatedly, Rödl (2003) claims that such a system is composed of sentences displaying categorical form: unlike hypothetical sentences, categorical sentences do not say what happens if such-and-such is the case, but say what happens period.

${ }^{5}$ For comments and suggestions I wish to thank Hanjo Glock, Mirjam Steudler and especially Philip Kitcher. 
Godfrey-Smith, P. (1999). Functions: Consensus Without Unity. In D. J. Buller (Ed.), Function, selection, and design, pp. 185-197. Albany, N.Y.: State University of New York Press.

Griffiths, P. E. (1993). Functional Analysis and Proper Functions. The British Journal for the Philosophy of Science 44(3), 409-422.

Jarosz, A. and A. Davelos (2006). Effects of Disease in Wild Plant Populations and the Evolution of Pathogen Aggressiveness. New Phytologist 129(3), 371-387.

Kitcher, P. (2003). Function and Design. In In Mendel's Mirror: Philosophical Reflections on Biology, pp. 159-176. Oxford: Oxford University Press.

Kitcher, P. (2011). The Ethical Project. Cambridge, MA: Harvard University Press. In press.

McLaughlin, P. (2001). What Functions Explain. Functional Explanation and Self-Reproducing Systems. Cambridge: Cambridge University Press.

Millikan, R. G. (1984). Language, Thought, and Other Biological Categories. Cambridge, Mass.: MIT Press.

Millikan, R. G. (2002). Biofunctions: Two Paradigms. In A. Ariew, R. Cummins, and M. Perlman (Eds.), Functions: New Essays in the Philosophy of Psychology and Biology, pp. 113-143. Oxford: Oxford University Press.

Neander, K. (1991). The Teleological Notion of 'Function'. Australasian Journal of Philosophy 69(4), 454-468.

Rödl, S. (2003). Norm und Natur. Deutsche Zeitschrift für Philosophie 51(1), 99-114.

Thompson, M. (2008). Life and Action. Elementary Structures of Practice and Practical Thought. Cambridge: Harvard University Press.

Weber, M. (2005). Holism, Coherence and the Dispositional Concept of Functions. Annals of the History and Philosophy of Biology 10, 189201.

Wright, L. (1973). Functions. The Philosophical Review 82(2), 139-168. 